\title{
Evidence for Enhanced Exosome Production in Aromatase Inhibitor-Resistant Breast Cancer Cells
}

\author{
Giuseppina Augimeri ${ }^{1, \dagger}$, Giusi La Camera ${ }^{1,+}$, Luca Gelsomino ${ }^{1}$, Cinzia Giordano ${ }^{1,2}$, \\ Salvatore Panza ${ }^{1}$, Diego Sisci ${ }^{1}{ }^{\mathbb{D}}$, Catia Morelli ${ }^{1}{ }^{1}$, Balázs Győrffy ${ }^{3,4}$, Daniela Bonofiglio ${ }^{1,2}{ }^{\mathbb{D}}$, \\ Sebastiano Andò ${ }^{1,2, \ddagger}$, Ines Barone ${ }^{1, *, \ddagger}$ (1) and Stefania Catalano ${ }^{1,2, *, \ddagger}$ \\ 1 Department of Pharmacy, Health and Nutritional Sciences, Via P Bucci, University of Calabria, \\ 87036 Arcavacata di Rende (CS), Italy; giusy.augimeri@gmail.com (G.A.); \\ giusylacamera93@gmail.com (G.L.C.); luca.gelsomino@unical.it (L.G.); cinzia.giordano@unical.it (C.G.); \\ sasapanza@libero.it (S.P.); dsisci@unical.it (D.S.); catia.morelli@unical.it (C.M.); \\ daniela.bonofiglio@unical.it (D.B.); sebastiano.ando@unical.it (S.A.) \\ 2 Centro Sanitario, Via P Bucci, University of Calabria, 87036 Arcavacata di Rende (CS), Italy \\ 3 Bioinformatics and 2nd Department of Pediatrics, Semmelweis University, 1094 Budapest, Hungary; \\ zsalab2@yahoo.com \\ 4 TTK Cancer Biomarker Research Group, 1117 Budapest, Hungary \\ * Correspondence: ines.barone@unical.it (I.B.); stefania.catalano@unical.it (S.C.); Tel.: +39-0984-496216 (I.B.); \\ +39-0984-496207 (S.C.) \\ + These authors contributed equally to this work. \\ $\ddagger$ Joint senior author.
}

Received: 13 June 2020; Accepted: 13 August 2020; Published: 14 August 2020

\begin{abstract}
Aromatase inhibitors (AIs) represent the standard anti-hormonal therapy for post-menopausal estrogen receptor-positive breast cancer, but their efficacy is limited by the emergence of $\mathrm{AI}$ resistance $\left(\mathrm{AI}^{\mathrm{R}}\right)$. Exosomes act as vehicles to engender cancer progression and drug resistance. The goal of this work was to study exosome contribution in $\mathrm{AI}^{\mathrm{R}}$ mechanisms, using estrogen-dependent MCF-7 breast cancer cells as models and MCF-7 LTED (Long-Term Estrogen Deprived) subline, modeling $\mathrm{AI}^{\mathrm{R}}$. We found that exosome secretion was significantly increased in MCF-7 LTED cells compared to MCF-7 cells. MCF-7 LTED cells also exhibited a higher amount of exosomal RNA and proteins than MCF-7 cells. Proteomic analysis revealed significant alterations in the cellular proteome. Indeed, we showed an enrichment of proteins frequently identified in exosomes in MCF-7 LTED cells. The most up-regulated proteins in MCF-7 LTED cells were represented by Rab GTPases, important vesicle transport-regulators in cancer, that are significantly mapped in "small GTPase-mediated signal transduction", "protein transport" and "vesicle-mediated transport" Gene Ontology categories. Expression of selected Rab GTPases was validated by immunoblotting. Collectively, we evidence, for the first time, that $\mathrm{AI}^{\mathrm{R}}$ breast cancer cells display an increased capability to release exosomes, which may be associated with an enhanced Rab GTPase expression. These data provide the rationale for further studies directed at clarifying exosome's role on endocrine therapy, with the aim to offer relevant markers and druggable therapeutic targets for the management of hormone-resistant breast cancers.
\end{abstract}

Keywords: breast cancer; endocrine resistance; exosomes; Rab GTPases

\section{Introduction}

According to the GLOBOCAN statistics in 2018, breast cancer represents the most commonly diagnosed cancer and the deadliest type of malignancy among the female population on a world scale, showing morbidity and mortality rates of $\sim 25 \%$ and $\sim 15 \%$, respectively [1]. Clinical decisions 
are generally dependent on disease stage and expression of estrogen (ER) and progesterone (PR) receptors, epidermal growth factor 2 receptor (HER2) and Ki-67. Hormone receptor-positive breast carcinomas account for almost $70-80 \%$ of all cancer cases and mainly overlap with luminal molecular subtypes [2]. In these tumors, endocrine-targeted treatments using aromatase inhibitors (AIs, i.e., letrozole, anastrozole and exemestane) represent the mainstay of the standard care both in the adjuvant and recurrent settings. Unfortunately, the development of "de novo" or acquired resistance to a prolonged estrogen withdrawal profoundly affects patient prognosis, which is a significant global concern [3]. To date, the hallmarks of hormonal resistance were studied thoroughly and may include the loss or mutations of ER, activation of growth factor signaling-dependent pathways, alterations of crucial cell cycle checkpoints, induction of epithelial-to-mesenchymal transition (EMT), cancer stem cell activity, and heterotypic cellular interactions within the tumour microenvironment [4-11]. However, despite major advances in our understanding of these molecular events, we are still unable to effectively treat hormone-resistant diseases, highlighting the need to explore novel, clinically relevant markers and therapeutic targets.

Exosomes are nanoscale membrane vesicles with a diameter of $20-200 \mathrm{~nm}$, belonging to the large family of the small extracellular vesicles (EVs), which are generated in the cells and released into the extracellular space. Although current separation and characterization methods for the EVs do not really allow us to define the specific subclass of EVs such as exosomes, as described in the "Minimal information for studies of extracellular vesicles 2018-(MISE2018)" [12], a large amount of literature in recent decades has highlighted the involvement of these small EVs in cancer biology. The attracted considerable interest in these vesicles relies on their ability to shuttle from one cell to another and deliver signals or messages to particular recipient cells. Indeed, they contain several biomolecules, such as nucleic acids, proteins, and lipids, that mediate crosstalk between neighboring or anatomically distant cells [13]. In recent years, it has been reported that the mechanisms of exosome biogenesis/release are deregulated in cancer, including breast cancer, with increased exosome counts in cancer cell lines and in patients' blood [14,15]. Indeed, breast cancer-derived exosomes, on the basis of their own cargo, may exhibit a wide array of biological activities, such as the induction of reactive oxygen species, autophagy and DNA damage repair response in normal human primary mammary epithelial cells [16], oncogenic transformation and tumor formation [17-19], impairment of the immune response [20,21], stimulation of mesenchymal stem cell differentiation into cancer-associated fibroblasts (CAFs) [22] along with increased fibroblast proliferation and lifespan [23]. On the other hand, only a few studies demonstrated the effect of the horizontal transfer of the resistance between cancer cells. For instance, tumor exosomes might contribute to chemotherapy failure by transferring functional p-glycoprotein from chemo-resistant MCF-7 cells [24], or interfere with the antineoplastic properties of Trastuzumab through different mechanisms [25-27]. Overexpression of the thymidine kinase 1 (TK1) and the cyclin-dependent kinase 9 (CDK9) in plasma-derived exosomes was significantly correlated with clinical resistance to CDK4/6 inhibitors in metastatic breast cancer patients [28]. In addition, exosomes from tamoxifen-resistant MCF-7 cells, by transfer miR-221/222 [29] or lncRNA UCA1 (Urothelial Carcinoma-Associated 1) [30], promoted the tamoxifen-resistant phenotype in MCF-7-sensitive cells. It has also been found that exosomes from a tamoxifen-resistant subline led to the irreversible cross-resistance of the parental MCF-7 cells to both tamoxifen and the anti-diabetic drug metformin [31]. Interestingly, the horizontal transfer of mitochondrial DNA from exosomes promoted an escape from dormancy in tamoxifen-resistant breast cancer cell lines [32]. Although it is becoming widely acknowledged to the scientific community the tumor-supportive role of the intercellular communications mediated by the secretion of exosomes from cancer cells, their involvement in the mechanisms underlying insensitivity to AI treatments has not been yet researched. In the present study, we show that resistance to AIs is associated to an enhanced exosome production, which appears to be related to an increased Rab GTPase expression. 


\section{Results}

\subsection{MCF-7 LTED (Long-Term Estrogen Deprived) Cells Exhibit Increased Exosome Production}

As experimental models to evaluate whether $\mathrm{AI}^{\mathrm{R}}$ may result in any changes in exosome production, we used parental human ER $\alpha$-positive MCF-7 breast cancer cells and MCF-7 LTED (Long-Term Estrogen Deprived) cells, which have gradually acquired estrogen independence after six-month culture in estrogen/steroid-free conditions, thereby modeling $\mathrm{AI}^{\mathrm{R}}$ [33]. In this cell line, well characterized for its resistant phenotype, we observed a low proliferation rate compared to their parental counterpart MCF-7 cells, having a doubling time of $54 \mathrm{~h}$ compared to $28 \mathrm{~h}$. First, we isolated exosomes from the conditioned medium of MCF-7 or MCF-7 LTED breast cancer cells cultured in the absence of serum to circumvent the collection of contaminating vesicles from FBS (Fetal Bovine Serum). Exosome isolation was performed by using a well-established ultracentrifugation scheme [34]. The obtained 100,000× $g$ pellet that represents the fraction of exosomes was characterized by transmission electron microscopy (TEM), immunoblot analysis and quantitative Nanoparticle Tracking Analysis (NTA). TEM images indicated that isolated vesicles were membrane-encapsulated particles with rounded shaped morphology, characteristic of exosomes (Figure 1a), while the identity of released exosomes was confirmed by the expression of classical exosomal markers, including Tumor susceptibility gene 101 (Tsg101), Alix and CD9 in exosome lysates (Figure 1b). As expected, the expression of the endoplasmic reticulum protein Calnexin was not detected in both samples (Figure 1b). In addition, NTA showed that the average size of exosomes seeded from MCF-7 (MCF-7-Exo) and MCF-7 LTED (LTED-Exo) cells was 127.6 \pm 2.9 and $141.7 \pm 1.6 \mathrm{~nm}$, respectively, highlighting that the majority of the purified particles were in the expected size range to be defined as exosomes (Figure 1c). Interestingly, when we analyzed the concentration of the secreted vesicles by using NTA, we found that the numbers of the exosomes (particles $/ \mathrm{mL} / 10^{6}$ cells) released in the conditioned medium of MCF-7 LTED cells increased about six-fold compared to those of exosomes isolated from parental cells $\left(6.09 \times 10^{10} \pm 0.48 \times 10^{10}\right.$ versus $\left.1.01 \times 10^{10} \pm 1.96 \times 10^{8}\right)$ (Figure 1c). We further determined the concentration of RNA and proteins within the exosomes and found similar results. Indeed, exosomes released from MCF-7 LTED cells exhibited a higher amount of RNA and proteins than those released from MCF-7 cells (Figure 1d,e). Moreover, since also apoptotic cells might increase the release of the extracellular vesicles displaying a broad size range, including exosomes, ectosomes/microvesicles and the larger "apoptotic body" [35], we tested if apoptosis might affect our results. We did not find any signs of apoptosis in MCF-7 and MCF-7 LTED cells, in the same experimental conditions used to obtain the exosome-enriched conditioned media, as demonstrated by the absence of changes in the internucleosomal fragmentation profile of genomic DNA, evaluated by TUNEL assay, and in the proteolysis of poly (ADP-ribose) polymerase (PARP), a known substrate of effector caspases (Supplementary Figure S1). Thus, exosome secretion was significantly increased in MCF-7 LTED cells compared to MCF-7 cells, further indicating that $\mathrm{AI}^{\mathrm{R}}$ phenotype might be associated with an enhanced capability of breast cancer cells to release exosomes.

\subsection{Quantitative Proteomic Analysis Shows Extensive Changes of Protein Expression in MCF-7 LTED Cells Compared with MCF-7 Cells}

To gain insight into the underlying molecular mechanisms responsible for the increased production of exosomes in MCF-7 LTED cells, quantitative proteome profiling of MCF-7 and MCF-7 LTED cells was carried out through label-free mass spectrometry using a nano-liquid chromatography coupled to an electrospray ionization-mass spectrometry (nLC-ESI-MS/MS). This comparison allowed the identification of 2794 differentially expressed proteins (FDR $<0.05$ ), of which 811 were significantly down-regulated and 795 significantly up-regulated in MCF-7 cells considering |1.5| fold as a cut-off. Thus, MCF-7 LTED cells have incurred extensive alterations in their proteome. A heatmap to display results of the supervised hierarchical clustering is shown in Figure 2. 
(a)
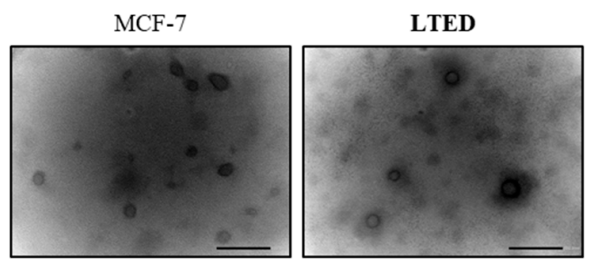

(c)
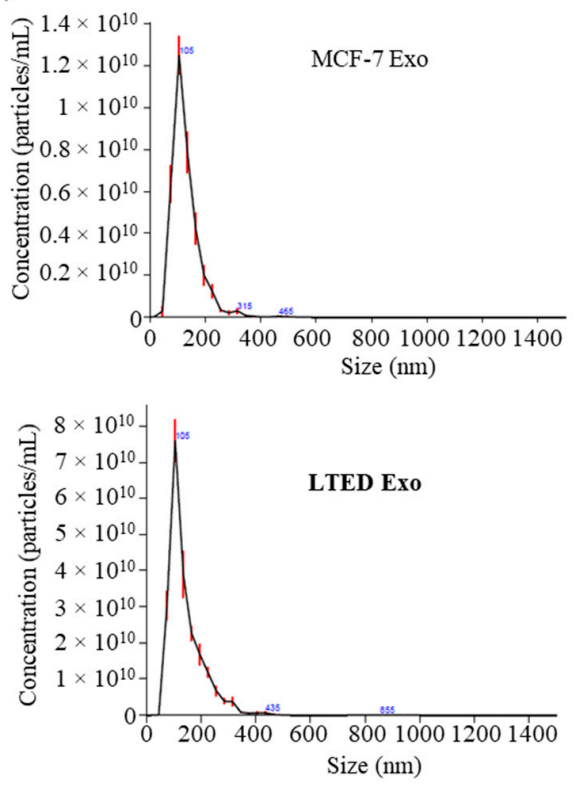

(d)

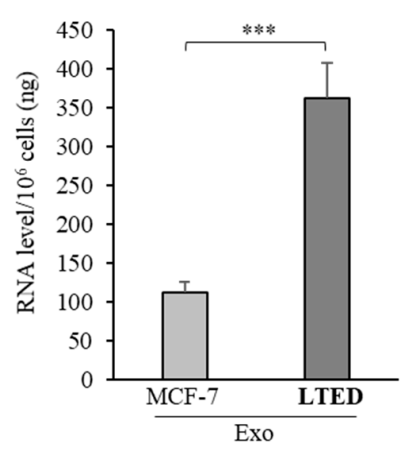

(b)

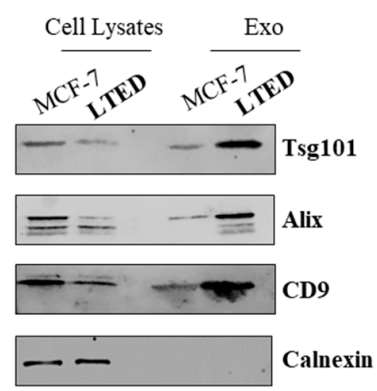

(e)

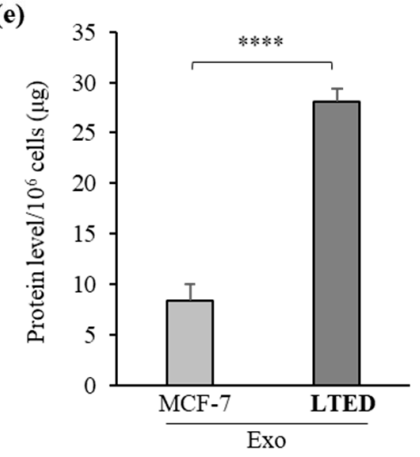

Figure 1. Increased release of exosomes from MCF-7 LTED cells compared to MCF-7 cells. (a) Representative micrograph of transmission electron microscopy (TEM) of exosomes from conditioned medium of MCF-7 (MCF-7 Exo) and MCF-7 LTED (LTED Exo) breast cancer cells. Scale bar, 100 nm; (b) Immunoblotting showing expression of the exosome hallmarks Tsg101, Alix and CD9 in equal amount $(4 \mu \mathrm{g})$ of exosome lysates (Exo) and whole cell lysates of MCF-7 and MCF-7 LTED cells. Calnexin was used to ensure that exosome samples were not contaminated with endoplasmic reticulum proteins; (c) Size distribution and concentration profiles of exosomes (Exo) recovered from MCF-7 and MCF-7 LTED breast cancer cell conditioned media (CM), measured by nanoparticle tracking analysis (NTA). The hystogram represents the mean \pm S.D. of exosome concentration (particles $/ \mathrm{mL} / 10^{6}$ cells) of 5 analyses; (d) Quantitation of average total amount of exosomal RNA per $10^{6}$ cells; (e) Quantitation of average total amount of exosomal proteins per $10^{6}$ cells. ${ }^{* *}, p<0.0005 ;{ }^{* * * *}, p<0.0001$. 

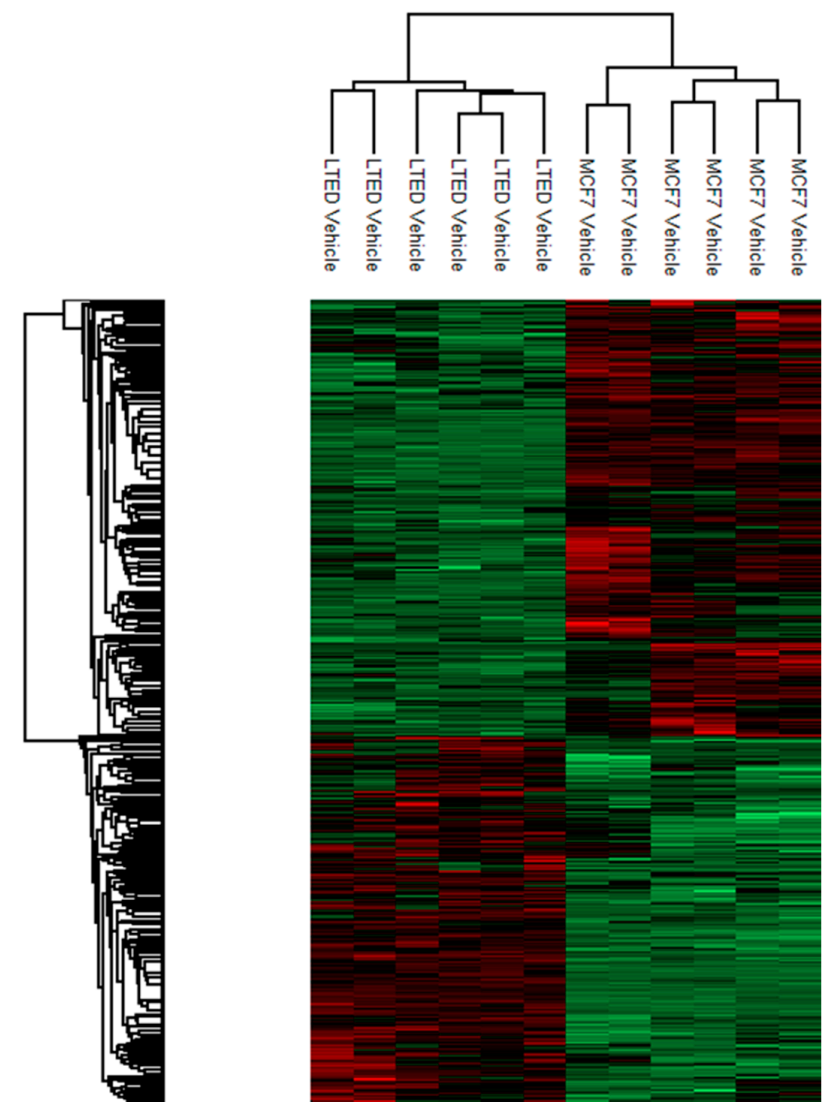

Figure 2. Heatmap representing supervised hierarchical clustering of the differentially expressed proteins in MCF-7 and MCF-7 LTED cells. Heatmap coding uses increasing brightness of red for degree of up-regulation and green for down-regulation. Black color stands for a median expression level.

These differentially expressed proteins were then subjected to Gene Ontology (GO) analysis to rank enriched biological processes (Table 1). Within this category, we found that that GO terms with the highest enrichment scores were related to "Translation" and "RNA splicing". Notably, in line with our previous findings, other high-ranking GO terms were related to "protein transport" and "vesicle-mediated transport".

In addition, referring to Exocarta (www.exocarta.org), a database for exosomal cargo, more than 50 proteins were detected in the proteomic profiles of both cell lines. Indeed, using the mean expression of all the genes as a signature, a significant enrichment of proteins that are more often identified in exosomes has been observed in MCF-7 LTED cells compared to MCF-7 cells (Figure 3).

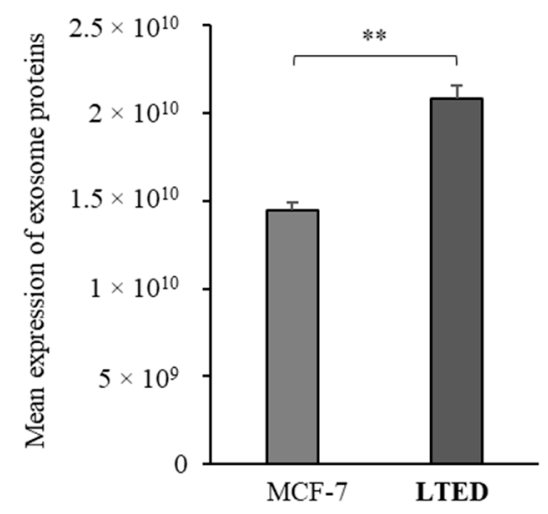

Figure 3. Differential expression of proteins identified in exosomes, revealed by Exocarta, using their mean expression as a signature. ${ }^{* *}, p<0.005$. 
Table 1. Top 10 biological processes identified by proteomic analysis in MCF-7 LTED cells compared to MCF-7 cells.

\begin{tabular}{lccccc}
\hline \multicolumn{1}{c}{ Term } & Count & $\mathbf{\%}$ & $p$ Value & Fold Enrichment & FDR \\
\hline RNA processing & 188 & 7.75 & $1.80 \times 10^{-31}$ & 2.33 & $3.35 \times 10^{-28}$ \\
Establishment of protein localization & 235 & 9.69 & $1.47 \times 10^{-30}$ & 2.07 & $2.73 \times 10^{-27}$ \\
Protein transport & 233 & 9.60 & $2.45 \times 10^{-30}$ & 2.07 & $4.56 \times 10^{-27}$ \\
Translation & 133 & 5.48 & $8.43 \times 10^{-30}$ & 2.72 & $1.57 \times 10^{-26}$ \\
Protein localization & 255 & 10.51 & $4.90 \times 10^{-29}$ & 1.96 & $9.13 \times 10^{-26}$ \\
RNA splicing & 118 & 4.86 & $5.10 \times 10^{-28}$ & 2.81 & $9.49 \times 10^{-25}$ \\
mRNA metabolic processing & 138 & 5.69 & $4.41 \times 10^{-27}$ & 2.53 & $8.20 \times 10^{-24}$ \\
Intracellular transport & 203 & 8.38 & $4.87 \times 10^{-27}$ & 2.09 & $9.07 \times 10^{-24}$ \\
mRNA processing & 123 & 5.07 & $2.14 \times 10^{-25}$ & 2.60 & $3.98 \times 10^{-22}$ \\
Vesicle-mediated transport & 168 & 6.92 & $1.39 \times 10^{-19}$ & 1.98 & $2.59 \times 10^{-16}$ \\
\hline
\end{tabular}

Differentially expressed proteins, determined by proteomic analysis, were analyzed to outline the most enriched biological processes. The table shows the top ten enriched terms that correspond to biological processes, along with the count of proteins involved, $p$ value, fold enrichment and False Discovery Rate (FDR).

\subsection{MCF-7 LTED Cells Show Enhanced Rab GTPase Protein Expression}

Proteomic analysis revealed multiple, functionally distinct proteins that are significantly altered in their expression among the two cell lines. Listed in Table 2 are proteins selected for either their large fold changes and perceived relevance for exosome production. Interestingly, the most up-regulated proteins in MCF-7 LTED cells were represented by Rab GTPases, important vesicle transport regulators in cancer, suggesting that MCF-7 LTED cells exhibited an enrichement of Rab GTPases. As expected, these proteins are significantly mapped in "small GTPase mediated signal transduction", "protein transport" and "vesicle-mediated transport" GO categories (Fold enrichment $=42.14$ and $p=2.55 \times 10^{-29}$, Fold enrichment $=16.9$ and $p=4.83 \times 10^{-22}$, Fold enrichment $=10.6$ and $p=5.13 \times 10^{-7}$, respectively).

To confirm the protein expression profile obtained in our proteomic analysis, we compared the expression of selected Rab GTPases, including Rab5, Rab7 and Rab11, for validation by immunoblotting. Results in Figure 4a showed an increase in the expression levels of Rab5 (approximately two-fold), Rab7 (approximately three-fold) and Rab 11 (approximately two-fold) in MCF-7 LTED cells compared to MCF-7 cells. Additionally, we performed real-time RT-PCR to analyze the mRNA expression levels of Rab GTPases. As shown in the Figure $4 b$, we found a significant increase in the mRNA levels for all the tested RAB genes in MCF-7 LTED cells compared to the parental MCF-7 cells, suggesting the existence of a possible regulation at transcriptional level of the $R A B$ gene expression in the $A I^{R}$ cells. Given the important role of Rab GTPases in modulating numerous steps of vesicle trafficking, these results may indicate that the increase in exosome production evidenced in MCF-7 LTED cells may be associated with an enhanced Rab GTPase protein expression. Subsequent studies will be aimed at assessing the role of specific Rab GTPases, the significance of the increased production of exosomes by aromatase inhibitor resistant cells along with any differences in their exosomal content. 
Table 2. Selected up-regulated proteins identified by proteomic analysis in MCF-7 LTED cells compared to MCF-7 cells (FDR < 0.05).

\begin{tabular}{|c|c|c|c|}
\hline Protein ID & Protein Name & Gene Names & Ratio AVG \\
\hline Q969Q5 & Ras-related protein Rab-24 & RAB24 & 6.41074493 \\
\hline Q6WKZ4 & Rab11 family-interacting protein 1 & RAB11FIP1 & 3.283109519 \\
\hline Q13637 & Ras-related protein Rab-32 & RAB32 & 3.134080381 \\
\hline A0A024R2K1 & Ras-related protein Rab-5A & RAB5A & 2.684524433 \\
\hline Q8WUD1 & Ras-related protein Rab-2B & $R A B 2 B$ & 2.358702001 \\
\hline A0A024RD41 & Ras-related protein Rab-23 & $R A B 23$ & 1.874367334 \\
\hline Q5HYI8 & Rab-like protein 3 & RABL3 & 1.832400186 \\
\hline Q9UL26 & Ras-related protein Rab-22A & $R A B 22 A$ & 1.72827705 \\
\hline Q6IQ22 & Ras-related protein Rab-12 & RAB12 & 1.608127936 \\
\hline A0A024RBA9 & Ras-related protein Rab-21 & $R A B 21$ & 1.583370444 \\
\hline A0A024R5J5 & Ras-related protein Rab-6A & $R A B 6 A$ & 1.470976838 \\
\hline A0A024R7V6 & Ras-related protein Rab-2A & $R A B 2 ; R A B 2 A$ & 1.455072096 \\
\hline Q9NP72 & Ras-related protein Rab-18 & RAB18 & 1.451684135 \\
\hline A0A024R7G2 & Ras-related protein Rab-3D & $R A B 3 D$ & 1.42786787 \\
\hline A0A024R7I7 & Ras-related protein Rab-3A & $R A B 3 A$ & 1.41943675 \\
\hline Q15907 & $\begin{array}{l}\text { Ras-related protein Rab-11B; } \\
\text { Ras-related protein Rab-11A }\end{array}$ & $R A B 11 B ; R A B 11 A$ & 1.403714286 \\
\hline A0A024R845 & Ras-related protein Rab-14 & RAB14 & 1.381289829 \\
\hline A0A024RB09 & Ras-related protein Rab-5B & $R A B 5 B$ & 1.333981668 \\
\hline A0A158RFU6 & Ras-related protein Rab-7a & $R A B 7 A$ & 1.332295086 \\
\hline O00194 & Ras-related protein Rab-27B & $R A B 27 B$ & 1.327797939 \\
\hline A0A024R1U4 & Ras-related protein Rab-5C & $R A B 5 C$ & 1.307181423 \\
\hline
\end{tabular}

The table shows differentially expressed proteins in MCF-7 and MCF-7 LTED cells, identified by proteomic analysis, along with the names of the corresponding gene and the ratio average between MCF-7 LTED and MCF-7 cells.

a)

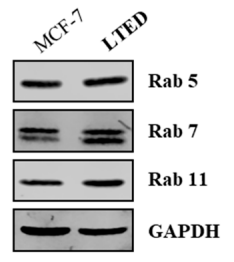

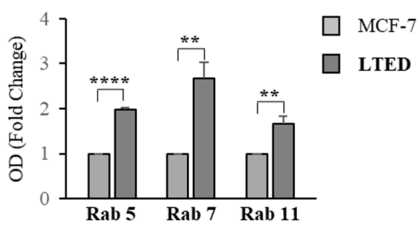

b)

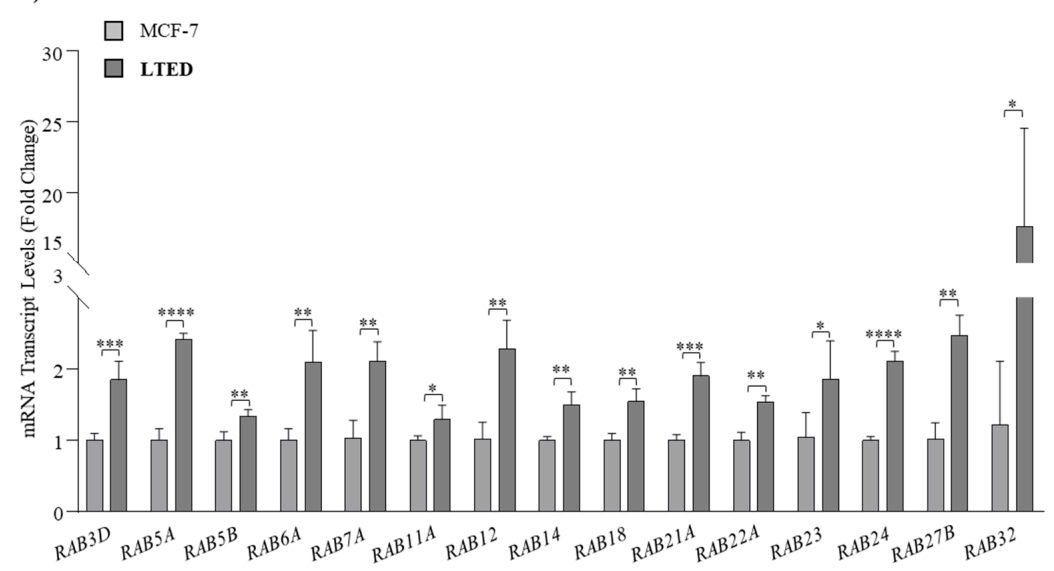

Figure 4. Rab expression in MCF-7 and MCF-7 LTED cells. (a) Immunoblot analysis showing protein levels of Rab5, Rab7 and Rab11 in MCF-7 and MCF-7 LTED cell lysates. GAPDH was used as a control for equal loading and transfer. The histogram represents the average fold change \pm S.D. of three separate experiments in which band intensities were evaluated in terms of optical density arbitrary units (OD), and expressed as fold change versus MCF-7 cells; (b) Real-time RT-PCR for mRNA levels of $R A B$ genes in MCF-7 and MCF-7-LTED cells. Data are expressed as means \pm S.D. of three different experiments, each performed in triplicate. ${ }^{*}, p<0.05 ;{ }^{* *} p<0.005 ;{ }^{* * *}, p<0.0005 ;{ }^{* * * *}, p<0.0001$. 


\section{Discussion}

Despite significant improvements in the treatment of ER-positive breast cancer following the introduction of AIs, "de novo" and acquired resistance is still an important concern clinically. For this reason, intensive research lines are currently underway and they are aimed at identifying further molecular markers and targets considering alterations in cancer proteomic profiles for more effective personalized therapies. In the present study, we show that resistance to AI treatments is associated to an enhanced exosome production, which appears to be related with an increased Rab GTPase protein expression.

Membrane trafficking machinery is characterized by a multifaceted network of signaling pathways able to connect various membrane-bound organelles of eukaryotic cells. Although each pathway is controlled by a specific set of components, they all enclose Rab GTPases that function as master regulators. Indeed, Rabs can virtually regulate all steps of membrane traffic from the formation of the transport vesicle at the donor membrane to its fusion at the target membrane. Rabs represent the largest family of small Ras-like GTPases with more than 70 members identified in humans that can be classified in several phylogenetic and functional groups [36]. They classically act as molecular switches by cycling between their active (GTP-bound) and inactive (GDP-bound) forms. The GTP-bound state can interact with several structurally and functionally effector proteins that select cargo, facilitate vesicle movement and verify the right site of fusion.

Although, in recent years, advanced progress toward understanding the regulation of exosome biogenesis and secretion by various Rab GTPases has been made, only a few Rab proteins have been shown to play a direct and significant role in these events. Particularly, Rab27A and Rab27B have been reported as the main proteins involved in the regulation of exosome release, while Rab9, Rab5 and Rab2, are generally associated with the endocytic pathway [37]. In addition, Rab11 and Rab35 have been demonstrated to regulate the recycling of membrane components from the endosomal compartment to the plasma membrane having a role in exosome production in different cell types [38]. Interestingly, we found by a quantitative proteomic analysis of the whole cell lysates of MCF-7 LTED (resistant) versus parental (sensitive) cells a significant increase in the expression of Rab GTPase proteins and identified "protein transport" and "vesicle-mediated transport" among the top ten enriched biological processes. Particularly, we found increased levels of Rab27B, Rab5 and Rab11 in terms of protein expression and mRNA content in $\mathrm{AI}^{\mathrm{R}}$ breast cancer cells. An increased expression of Rab GTPases in our resistant breast cancer cell model well fits with several findings reporting that these important vesicle transport regulators play essential roles in several cancer types [39-43], including breast cancers.

For instance, gene amplification and overexpression of Rab2A promoted breast cancer stem cell expansion via Erk1/2 activation and was associated with poor clinical outcome in patients with breast carcinoma [44]. Rab5A protein expression was correlated to enhanced migration of breast cancer cells and lymphatic dissemination in human breast cancer specimens $[45,46]$. More recently, a function of Rab7 in the proliferation, invasion, and xenograft tumor development of breast cancer cells was also reported [47], while Rab11, has been demonstrate to contributes to breast cancer cell invasion through trafficking of the $\alpha 6 \beta 4$ Integrin [48]. Moreover, Rabs have been found to participate to the intercellular communication between cancerous and stromal cells within the tumor microenvironment [49] and to the development of drug resistance. In this context, Rab27-dependent secretion of exosomes and metalloproteinases resulted into the mobilization of a pro-tumoral neutrophil population, thus supporting growth of a mouse mammary tumor and its lung dissemination [50]. Exosome shuttling between tumor-associated macrophages and cancer cells has been shown to be modulated by Rab27A/B and this mechanism has been involved to chemotherapy resistance [51]. Another Rab protein conveying resistance to a chemotherapeutic drug is the secretory Rab8, through an increased secretion of the cisplatin-resistance-associated protein TMEM205 (transmembrane protein 205) [52]. Moreover, STAT3/Rabs-mediated exosome release was correlated with a more aggressive and chemoresistant cancer phenotype under hypoxic conditions [53] and a recent study demonstrated a role for Rab18 in resistance to cisplatin-induced apoptosis [54]. 
In $\mathrm{AI}^{\mathrm{R}}$ breast cancer cells, we found an increased capability to release exosomes as revealed by Nanoparticle Tracking Analysis, which may be associated with the enhanced Rab GTPase expression. Concomitantly, exosomes released from resistant cells exhibited a higher amount of RNA and proteins than those secreted by parental cells. Moreover, based on Exocarta database, we also found in $\mathrm{AI}^{\mathrm{R}}$ breast cancer cells a significant enrichment of proteins that are often identified in exosomes.

\section{Materials and Methods}

\subsection{Antibodies}

Human anti-RAB5, RAB7, and RAB11 antibodies (Rab Family Antibody Sampler Kit \#9385) were acquired from Cell Signaling Technology (Danvers, MA, USA); human anti-GAPDH (sc-47724), anti-Calnexin (sc-11397) and anti-PARP (sc-7150) antibodies were from Santa Cruz Biotechnology (Dallas, TX, USA); human anti-Tsg101 (\#MA1-23296) antibody was from Invitrogen (Carlsbad, CA, USA); anti-Alix (ab186429) and anti-CD9 (ab92726) antibodies were acquired from Abcam (Cambridge, UK).

\subsection{Cell Cultures}

Human breast cancer cell line MCF-7 was from American Type Culture Collection, stored and authenticated according to supplier's instructions. MCF-7 cells were cultured in DMEM medium, containing $10 \% \mathrm{FBS}, 1 \%$ L-glutamine and $1 \mathrm{mg} / \mathrm{mL}$ penicillin-streptomycin at $37{ }^{\circ} \mathrm{C}$ with $5 \% \mathrm{CO}_{2}$ air. Long-term estrogen deprived (LTED) cells were derived from MCF-7 cells after estrogen-deprivation for six months [33]. MCF-7 LTED cells were grown in DMEM medium supplemented with 10\% Dextran-Coated Charcoal (Sigma-Aldrich, Milano, Italy), 1\% L-glutamine and $1 \mathrm{mg} / \mathrm{mL}$ penicillin-streptomycin. All cell lines, were regularly tested for morphology, doubling times, estrogen sensitivity and mycoplasma-negativity (MycoAlert Mycoplasma Detection Assay, Lonza, Basilea, $\mathrm{CH}$, Switzerland).

\subsection{Isolation of Tumor-Derived Exosomes}

MCF-7 and MCF-7 LTED cells were plated at a density of $3.5 \times 10^{6}$ cells $/ 75 \mathrm{~cm}^{2}$ flask in $10 \mathrm{~mL}$ of complete medium for $24 \mathrm{~h}$ and then incubated in serum-free medium. At least 5 flasks/conditions were used. After $48 \mathrm{~h}$, conditioned medium was harvested and exosomes were isolated by differential ultracentrifugation method [34]. Briefly, the first step was designed to eliminate large dead cells and cell debris by successive centrifugations at increasing speed (300 $\mathrm{g}$ and $2000 \times g$ for $10 \mathrm{~min}$, respectively). At each of these steps, the pellet was thrown away, and the supernatant was used to following step. The resulting supernatant was centrifuged at $10,000 \times g$ for $30 \mathrm{~min}$ to remove microvesicles and the final supernatant was then ultracentrifuged at $100,000 \times g$ for 70 min (Sorvall WX Ultra Series Centrifuge, T-865, Thermo Fisher Scientific, Milan, Italy). The obtained pellet, that corresponds to exosome, was washed in a large volume of PBS $(5 \mathrm{~mL})$ to eliminate any contaminating proteins and ultracentrifuged one last time at the same high speed. All steps were carried out at $4{ }^{\circ} \mathrm{C}$. The final exosome pellet was resuspended in PBS and stored at $-80^{\circ} \mathrm{C}$ until use [55].

\subsection{Transmission Electron Microscopy (TEM)}

Whole exosome extracts were fixed in $2 \%$ glutaraldehyde and then absorbed onto formovar-coated grids for $20 \mathrm{~min}$ in a dry environment. The grids were examined in a Jeol JEM 1400 Plus electron microscope (JEOL USA, Inc., MA, USA) at $80 \mathrm{kV}$.

\subsection{Immunoblot Analysis}

Cells and exosomes were lysed in RIPA Buffer (50 mM Tris-HCl, $150 \mathrm{mM} \mathrm{NaCl}, 1 \%$ Nonidet P-40, $0.5 \%$ sodium deoxycholate, $2 \mathrm{mM}$ sodium fluoride, $2 \mathrm{mM}$ EDTA, and $0.1 \%$ SDS) containing a mixture of protease inhibitors (aprotinin, phenylmethylsulfonyl fluoride, and sodium orthovanadate) for protein extraction. Equal amounts of cell and exosome extracts were resolved by SDS-PAGE as 
described in [56,57]. Images were acquired by Odissey FC (Licor, Lincoln, NE, USA) and the bands of interest were quantified using Scion Image laser densitometry scanning program (National Institutes of Health, MD, USA).

\subsection{Nanoparticle Tracking Analysis (NTA)}

Exosomes from MCF-7 and MCF-7 LTED cells were diluted in PBS before Nanoparticle Tracking Analysis (NTA), which measures the concentration and the size distribution of exosome in the $10 \mathrm{~nm}$ to $2 \mu \mathrm{m}$ range. NTA was undertaken using the NanoSight NS300 technology (Malvern Panalytical Ltd., Malvern, UK) equipped with a $488 \mathrm{~nm}$ laser that allows the tracking of both light scattering and Brownian motion of nanoparticles in a liquid suspension on a particle-by-particle basis. The assay was performed according to the recommendation of the instrument's manufacturer. Since NTA is more accurate between particle concentrations in the range of $2 \times 10^{8}$ to $2 \times 10^{9}$, esosomes from MCF-7 and MCF-7 LTED cells were diluted before analysis at 1:200 and 1:800, respectively. Briefly, sixty-second videos were recorded in five replicates for sample with optimized set parameters (the detection threshold was set to 5 for both samples). Data capture and further analysis were performed using the NTA software version 3.3 (Malvern Panalytical, LTD., Malvern, UK). Size distribution and concentration profiles were averaged across replicates to derive the presented results.

\subsection{RNA Extraction and Real-Time RT-PCR Assays}

Total RNA was extracted from exosomes using Total Exosome RNA and Protein Isolation Kit following the manufacturer's instructions (Thermo Fisher Scientific, Milan, Italy). Total cellular RNA was extracted using TRIZOL reagent (Life Technologies, Milan, Italy) as suggested by the manufacturer. Rab gene levels were measured by real-time RT-PCR, using SYBR Green Universal PCR Master Mix (Bio-Rad, Segrate, Italy) as previously described [58]. mRNA expression levels of genes were normalized on 18s mRNA content, and relative gene expression levels were calculated as described [59]. Primers are listed in Supplementary Table S1.

\subsection{TUNEL Assay}

Apoptosis was determined by enzymatic labeling of DNA strand breaks using terminal deoxynucleotidyl transferase-mediated deoxyuridine triphosphate nick end labeling, using APO-BrdUTM TUNEL Assay Kit (Promega, Madison, WI, USA) as described [60].

\subsection{Proteomic Analysis}

\subsubsection{Protein Digestion for MS Analysis}

For label-free quantitative proteomic analysis, MCF-7 and MCF-7 LTED cells were lysed with UA buffer (100 mM Tris $\mathrm{HCl}, \mathrm{pH}$ 8.5, and $8 \mathrm{M}$ urea) and the total concentration of proteins in solution was measured by Bicinchoninic acid (BCA, Thermo Fisher Scientific, Milan, Italy) assay. Next, $50 \mu \mathrm{g}$ of lysate was in-solution digested [61]. Briefly, proteins were reduced by TCEP, alkylated by chloroacetamide, and digested by Lys-C and trypsin, then peptides were desalted on StageTip C18 [62].

\subsubsection{Mass Spectrometry Analysis}

Each sample was analyzed as technical duplicate on a LC-ESI-MS-MS quadrupole Orbitrap QExactive-HF mass spectrometer (Thermo Fisher Scientific, Milan, Italy). Separation of peptides was achieved on a linear gradient from $93 \%$ solvent $\mathrm{A}(2 \% \mathrm{ACN}, 0.1 \%$ formic acid) to $60 \%$ solvent B (80\% acetonitrile, $0.1 \%$ formic acid) over $110 \mathrm{~min}$, and from $60 \%$ to $100 \%$ solvent $\mathrm{B}$ in $10 \mathrm{~min}$ at a constant flow rate of $0.25 \mu \mathrm{L} / \mathrm{min}$ on UHPLC Easy-nLC 1000 (Thermo Fischer Scientific, Milan, Italy) connected to a $23 \mathrm{~cm}$ fused-silica emitter of $75 \mu \mathrm{m}$ ID (New Objective, Inc. Woburn, MA, USA), packed in-house with ReproSil-Pur C18-AQ $1.9 \mu \mathrm{m}$ beads (Dr Maisch Gmbh, Ammerbuch, Germany) using a high-pressure bomb loader (Proxeon, Odense, Denmark). MS data were acquired using a 
data-dependent top 20 method for HCD fragmentation. Survey full scan MS spectra (300-1650 Th) were acquired in the Orbitrap with resolution 60,000, AGC target 3e6, IT 20 ms. For HCD spectra, resolution was set to 15,000 at $\mathrm{m} / \mathrm{z} 200$, AGC target 1e5, IT $80 \mathrm{~ms}$; NCE 28\%, dynamic exclusion $20 \mathrm{~s}$ and isolation width $1.2 \mathrm{~m} / \mathrm{z}$ [63].

\subsubsection{MS Analysis}

Raw files were processed via MaxQuant software 1.5.2.8 (Computational Systems Biochemistry Martinsried, Germany) [64], with Andromeda search engine [65]. MS/MS peak lists were searched against the database Uniprot_cp_Human, setting trypsin specificity and up to two missed cleavages; cysteine carbamidomethyl as fixed modification, methionine oxidation and protein N-terminal acetylation as variable modifications. Mass deviation for MS-MS peaks was set at $20 \mathrm{ppm}$. The peptides and protein FDR were set to 0.01 ; the minimal length required for a peptide was six amino acids; a minimum of two peptides and at least one unique peptide were required for high-confidence protein identification. Proteins were analyzed in a label-free manner, using protein intensity values normalized across the entire data set. Each protein was assigned to the functional classification based on the Gene Ontology annotation system using the Database for Annotation, Visualization and Integrated Discovery version 6.8 (https://david.ncifcrf.gov/). The mass spectrometry proteomic data have been deposited to the ProteomeXchange Consortium via PRIDE partner repository with the dataset identifier PXD012431.

\subsection{Statistical Analysis}

Data were analyzed for statistical significance using a two-tailed Student's Test and GraphPad-Prism 7 (GraphPad Inc., CA, USA). Standard deviations/S.D. are shown. Statistical analysis for proteomic analysis was performed via Perseus platform version 1.5.1.6 (Computational Systems Biochemistry, Martinsried, Germany) on Normalized Intensities by $z$-score normalization, using $t$-test, Permutation test and FDR 0.05; statistically significant proteins were submitted to Hierarchical Clustering analysis and represented on HeatMaps. In the gene ontology analysis, only categories containing at least three genes and those having a Benjamini-corrected $p$ value below 0.05 were accepted as significant.

\section{Conclusions}

Evidence for the functions and roles of exosomes as mediators of therapy failure is growing, and recent studies have detected more exosome-releasing properties in drug-resistant settings [66]. To our knowledge, this is the first study reporting that the $\mathrm{AI}^{\mathrm{R}}$ phenotype might be associated with an increased capability of breast cancer cells to release exosomes, raising the need to better evaluate the impact of exosomes on endocrine therapy. Therefore, based on our data, it will be intriguing and worthwhile to further investigate this mechanism in future studies aimed at clarifying: (i) which Rab GTPase may be primarily involved in exosome-mediated $\mathrm{AI}^{\mathrm{R}}$ in breast cancer; (ii) which specific molecules delivered by exosomes may cause extrinsic therapy resistance; (iii) which recipient cells, such as non-resistant cancer cells, macrophages, fibroblasts, etc., can be driven by exosomal molecules from resistant cells within the "AI ${ }^{\mathrm{R}}$ microenvironment". Unraveling these events implies several clinical implications prospectively. First, because exosomes contain proteins, RNA, and many types of miRNAs whose levels can be measured in blood, urine, or other bodily fluids, their evaluation might offer new predictive markers for hormonal response. Second, a deeper understanding of the key molecules involved in $\mathrm{AI}^{\mathrm{R}}$ vesiculation may help to identify potential therapeutic targets, which may be useful to extend the duration of sensitivity to estrogen deprivation, or to overwhelm resistance at its time of emergence in breast cancer patients.

Supplementary Materials: Supplementary materials can be found at http:/www.mdpi.com/1422-0067/21/16/ 5841/s1, Figure S1: Evaluation of apoptosis in MCF-7 and MCF-7 LTED cells, Table S1: Oligonucleotide primers used in this study. 
Author Contributions: Conceptualization, S.A., I.B. and S.C.; methodology, G.A., G.L.C., L.G., S.P. and C.M.; validation, C.G., D.S. and D.B.; formal analysis, I.B., S.C. and B.G.; investigation, G.A., I.B. and G.L.C.; data curation, D.S. and D.B.; writing—original draft preparation, I.B., G.A. and G.L.C.; writing-review and editing, S.A., I.B. and S.C.; supervision, I.B. and S.C.; funding acquisition, S.A., I.B. and S.C. All authors have read and agreed to the published version of the manuscript.

Funding: This research was funded by My First AIRC Grant (MFAG) \#16899 and BANDO PRIN 2017 \#2017WNKSLR_005 to I. Barone; by AIRC Investigator Grant (IG) \#18602 to S. Andò; by AIRC Investigator Grant (IG) \#21414 and BANDO PRIN 2017 \#2017EKMFTN_001 to S. Catalano; by the Higher Education Institutional Excellence Programme of the Ministry for Innovation and Technology in Hungary, within the framework of the Bionic thematic programme of the Semmelweis University to B. Győrffy.

Acknowledgments: MS-Proteomics analysis was performed at the Cogentech Proteomics/MS Facility, Milan, Italy.

Conflicts of Interest: The authors declare no conflict of interest.

\section{References}

1. Bray, F.; Ferlay, J.; Soerjomataram, I.; Siegel, R.L.; Torre, L.A.; Jemal, A. Global cancer statistics 2018: GLOBOCAN estimates of incidence and mortality worldwide for 36 cancers in 185 countries. CA Cancer J. Clin. 2018, 68, 394-424. [CrossRef]

2. Perou, C.M.; Sorlie, T.; Eisen, M.B.; van de Rijn, M.; Jeffrey, S.S.; Rees, C.A.; Pollack, J.R.; Ross, D.T.; Johnsen, H.; Akslen, L.A.; et al. Molecular portraits of human breast tumours. Nature 2000, 406, 747-752. [CrossRef]

3. Murphy, C.G.; Dickler, M.N. Endocrine resistance in hormone-responsive breast cancer: Mechanisms and therapeutic strategies. Endocr. Relat. Cancer 2016, 23, R337-R352. [CrossRef] [PubMed]

4. Martin, L.-A.; Farmer, I.; Johnston, S.R.D.; Ali, S.; Dowsett, M. Elevated ERK1/ERK2/estrogen receptor cross-talk enhances estrogen-mediated signaling during long-term estrogen deprivation. Endocr. Relat. Cancer 2005, 12, S75-S84. [CrossRef] [PubMed]

5. Sabnis, G.J.; Jelovac, D.; Long, B.; Brodie, A. The Role of Growth Factor Receptor Pathways in Human Breast Cancer Cells Adapted to Long-term Estrogen Deprivation. Cancer Res. 2005, 65, 3903-3910. [CrossRef] [PubMed]

6. Santen, R.J.; Song, R.X.; Zhang, Z.; Kumar, R.; Jeng, M.H.; Masamura, A.; Lawrence, J., Jr.; Berstein, L.; Yue, W. Long-term estradiol deprivation in breast cancer cells up-regulates growth factor signaling and enhances estrogen sensitivity. Endocr. Relat. Cancer 2005, 12, S61-S73. [CrossRef] [PubMed]

7. Barone, I.; Iacopetta, D.; Covington, K.R.; Cui, Y.; Tsimelzon, A.; Beyer, A.; Andò, S.; Fuqua, S.A.W.; Anna, T. Phosphorylation of the mutant K303R estrogen receptor $\alpha$ at serine 305 affects aromatase inhibitor sensitivity. Oncogene 2010, 29, 2404-2414. [CrossRef]

8. Chen, S. An "Omics" Approach to Determine the Mechanisms of Acquired Aromatase Inhibitor Resistance. Omics A J. Integr. Biol. 2011, 15, 347-352. [CrossRef]

9. Thangavel, C.; Dean, J.L.; Ertel, A.; Knudsen, K.E.; Aldaz, C.M.; Witkiewicz, A.K.; Clarke, R.; Knudsen, E.S. Therapeutically activating RB: Reestablishing cell cycle control in endocrine therapy-resistant breast cancer. Endocr. Relat. Cancer 2011, 18, 333-345. [CrossRef]

10. Dittmer, J.; Leyh, B. The impact of tumor stroma on drug response in breast cancer. Semin. Cancer Biol. 2015, 31, 3-15. [CrossRef]

11. Ma, C.X.; Reinert, T.; Chmielewska, I.; Ellis, M.J. Mechanisms of aromatase inhibitor resistance. Nat. Rev. Cancer 2015, 15, 261-275. [CrossRef] [PubMed]

12. Théry, C.; Witwer, K.W.; Aikawa, E.; Alcaraz, M.J.; Anderson, J.D.; Andriantsitohaina, R.; Antoniou, A.; Arab, T.; Archer, F.; Atkin-Smith, G.K.; et al. Minimal information for studies of extracellular vesicles 2018 (MISEV2018): A position statement of the International Society for Extracellular Vesicles and update of the MISEV2014 guidelines. J. Extracell. Vesicles 2018, 7, 1535750. [CrossRef] [PubMed]

13. Raposo, G.; Stoorvogel, W. Extracellular vesicles: Exosomes, microvesicles, and friends. J Cell Biol 2013, 200, 373-383. [CrossRef] [PubMed]

14. Khan, S.; Bennit, H.F.; Turay, D.; Perez, M.; Mirshahidi, S.; Yuan, Y.; Wall, N.R. Early diagnostic value of survivin and its alternative splice variants in breast cancer. BMC Cancer 2014, 14, 176. [CrossRef]

15. Bebelman, M.P.; Smit, M.J.; Pegtel, D.M.; Baglio, S.R. Biogenesis and function of extracellular vesicles in cancer. Pharm. Ther. 2018, 188, 1-11. [CrossRef] 
16. Dutta, S.; Warshall, C.; Bandyopadhyay, C.; Dutta, D.; Chandran, B. Interactions between exosomes from breast cancer cells and primary mammary epithelial cells leads to generation of reactive oxygen species which induce DNA damage response, stabilization of p53 and autophagy in epithelial cells. PLoS ONE 2014, 9, e97580. [CrossRef]

17. Yu, J.L.; May, L.; Lhotak, V.; Shahrzad, S.; Shirasawa, S.; Weitz, J.I.; Coomber, B.L.; Mackman, N.; Rak, J.W. Oncogenic events regulate tissue factor expression in colorectal cancer cells: Implications for tumor progression and angiogenesis. Blood 2005, 105, 1734-1741. [CrossRef]

18. Antonyak, M.A.; Li, B.; Boroughs, L.K.; Johnson, J.L.; Druso, J.E.; Bryant, K.L.; Holowka, D.A.; Cerione, R.A. Cancer cell-derived microvesicles induce transformation by transferring tissue transglutaminase and fibronectin to recipient cells. Proc. Natl. Acad. Sci. USA 2011, 108, 4852-4857. [CrossRef]

19. Melo, S.A.; Sugimoto, H.; O'Connell, J.T.; Kato, N.; Villanueva, A.; Vidal, A.; Qiu, L.; Vitkin, E.; Perelman, L.T.; Melo, C.A.; et al. Cancer exosomes perform cell-independent microRNA biogenesis and promote tumorigenesis. Cancer Cell 2014, 26, 707-721. [CrossRef]

20. Chow, A.; Zhou, W.; Liu, L.; Fong, M.Y.; Champer, J.; Van Haute, D.; Chin, A.R.; Ren, X.; Gugiu, B.G.; Meng, Z.; et al. Macrophage immunomodulation by breast cancer-derived exosomes requires Toll-like receptor 2-mediated activation of NF-kB. Sci. Rep. 2014, 4, 5750. [CrossRef]

21. Kenific, C.M.; Nogués, L.; Lyden, D. Pre-Metastatic Niche Formation Has Taken Its TOLL. Cancer Cell 2016, 30, 189-191. [CrossRef]

22. Cho, J.A.; Park, H.; Lim, E.H.; Lee, K.W. Exosomes from breast cancer cells can convert adipose tissue-derived mesenchymal stem cells into myofibroblast-like cells. Int. J. Oncol. 2011, 40, 130-138. [CrossRef]

23. Gutkin, A.; Uziel, O.; Beery, E.; Nordenberg, J.; Pinchasi, M.; Goldvaser, H.; Henick, S.; Goldberg, M.; Lahav, M. Tumor cells derived exosomes contain hTERT mRNA and transform nonmalignant fibroblasts into telomerase positive cells. Oncotarget 2016, 7, 59173-59188. [CrossRef]

24. Lv, M.M.; Zhu, X.Y.; Chen, W.X.; Zhong, S.L.; Hu, Q.; Ma, T.F.; Zhang, J.; Chen, L.; Tang, J.H.; Zhao, J.H. Exosomes mediate drug resistance transfer in MCF-7 breast cancer cells and a probable mechanism is delivery of P-glycoprotein. Tumor Biol 2014, 35, 10773-10779. [CrossRef]

25. Martinez, V.G.; O’Neill, S.; Salimu, J.; Breslin, S.; Clayton, A.; Crown, J.; O’Driscoll, L. Resistance to HER2-targeted anti-cancer drugs is associated with immune evasion in cancer cells and their derived extracellular vesicles. Oncoimmunology 2017, 6, e1362530. [CrossRef]

26. Dong, H.; Wang, W.; Chen, R.; Zhang, Y.; Zou, K.; Ye, M.; He, X.; Zhang, F.; Han, J. Exosome-mediated transfer of lncRNA-SNHG14 promotes trastuzumab chemoresistance in breast cancer. Int. J. Oncol. 2018, 53, 1013-1026. [CrossRef]

27. Han, M.; Hu, J.; Lu, P.; Cao, H.; Yu, C.; Li, X.; Qian, X.; Yang, X.; Yang, Y.; Han, N.; et al. Exosome-transmitted miR-567 reverses trastuzumab resistance by inhibiting ATG5 in breast cancer. Cell Death Dis. 2020, 11, 43. [CrossRef]

28. Del Re, M.; Bertolini, I.; Crucitta, S.; Fontanelli, L.; Rofi, E.; De Angelis, C.; Diodati, L.; Cavallero, D.; Gianfilippo, G.; Salvadori, B.; et al. Overexpression of TK1 and CDK9 in plasma-derived exosomes is associated with clinical resistance to CDK4/6 inhibitors in metastatic breast cancer patients. Breast Cancer Res. Treat. 2019, 178, 57-62. [CrossRef]

29. Wei, Y.; Lai, X.; Yu, S.; Chen, S.; Ma, Y.; Zhang, Y.; Li, H.; Zhu, X.; Yao, L.; Zhang, J. Exosomal miR-221/222 enhances tamoxifen resistance in recipient ER-positive breast cancer cells. Breast Cancer Res. Treat. 2014, 147, 423-431. [CrossRef] [PubMed]

30. Xu, C.G.; Yang, M.F.; Ren, Y.Q.; Wu, C.H.; Wang, L.Q. Exosomes mediated transfer of lncRNA UCA1 results in increased tamoxifen resistance in breast cancer cells. Eur. Rev. Med. Pharm. Sci. 2016, 20, 4362-4368.

31. Semina, S.E.; Scherbakov, A.M.; Vnukova, A.A.; Bagrov, D.V.; Evtushenko, E.G.; Safronova, V.M.; Golovina, D.A.; Lyubchenko, L.N.; Gudkova, M.V.; Krasil'nikov, M.A. Exosome-Mediated Transfer of Cancer Cell Resistance to Antiestrogen Drugs. Molecules 2018, 23, 829. [CrossRef]

32. Sansone, P.; Savini, C.; Kurelac, I.; Chang, Q.; Amato, L.B.; Strillacci, A.; Stepanova, A.; Iommarini, L.; Mastroleo, C.; Daly, L.; et al. Packaging and transfer of mitochondrial DNA via exosomes regulate escape from dormancy in hormonal therapy-resistant breast cancer. Proc. Natl. Acad. Sci. USA 2017, 114, E9066-E9075. [CrossRef] 
33. Lombardo, Y.; Faronato, M.; Filipovic, A.; Vircillo, V.; Magnani, L.; Coombes, R.C. Nicastrin and Notch4 drive endocrine therapy resistance and epithelial to mesenchymal transition in MCF7 breast cancer cells. Breast Cancer Res. 2014, 16, R62. [CrossRef] [PubMed]

34. Thery, C.; Amigorena, S.; Raposo, G.; Clayton, A. Isolation and characterization of exosomes from cell culture supernatants and biological fluids. Curr. Protoc. Cell. Biol. 2006, 30, 3.22.1-3.22.29. [CrossRef]

35. Lynch, C.; Panagopoulou, M.; Gregory, C.D. Extracellular Vesicles Arising from Apoptotic Cells in Tumors: Roles in Cancer Pathogenesis and Potential Clinical Applications. Front. Immunol. 2017, 8, 1174. [CrossRef]

36. Pereira-Leal, J.B.; Seabra, M.C. Evolution of the Rab family of small GTP-binding proteins. J. Mol. Biol. 2001, 313, 889-901. [CrossRef]

37. Ostrowski, M.; Carmo, N.B.; Krumeich, S.; Fanget, I.; Raposo, G.; Savina, A.; Moita, C.F.; Schauer, K.; Hume, A.N.; Freitas, R.P.; et al. Rab27a and Rab27b control different steps of the exosome secretion pathway. Nat. Cell Biol. 2010, 12, 19-30. [CrossRef]

38. Blanc, L.; Vidal, M. New insights into the function of Rab GTPases in the context of exosomal secretion. Small Gtpases 2018, 9, 95-106. [CrossRef]

39. Thomas, J.D.; Zhang, Y.J.; Wei, Y.H.; Cho, J.H.; Morris, L.E.; Wang, H.Y.; Zheng, X.F. Rab1A is an mTORC1 activator and a colorectal oncogene. Cancer Cell 2014, 26, 754-769. [CrossRef]

40. Xu, B.H.; Li, X.X.; Yang, Y.; Zhang, M.Y.; Rao, H.L.; Wang, H.Y.; Zheng, X.F. Aberrant amino acid signaling promotes growth and metastasis of hepatocellular carcinomas through Rab1A-dependent activation of mTORC1 by Rab1A. Oncotarget 2015, 6, 20813-20828. [CrossRef]

41. Bao, Z.S.; Li, M.Y.; Wang, J.Y.; Zhang, C.B.; Wang, H.J.; Yan, W.; Liu, Y.W.; Zhang, W.; Chen, L.; Jiang, T. Prognostic value of a nine-gene signature in glioma patients based on mRNA expression profiling. CNS Neurosci. Ther. 2014, 20, 112-118. [CrossRef] [PubMed]

42. Abd Elmageed, Z.Y.; Yang, Y.; Thomas, R.; Ranjan, M.; Mondal, D.; Moroz, K.; Fang, Z.; Rezk, B.M.; Moparty, K.; Sikka, S.C.; et al. Neoplastic reprogramming of patient-derived adipose stem cells by prostate cancer cell-associated exosomes. Stem Cells 2014, 32, 983-997. [CrossRef] [PubMed]

43. Tzeng, H.T.; Wang, Y.C. Rab-mediated vesicle trafficking in cancer. J. Biomed. Sci. 2016, 23, 70. [CrossRef]

44. Luo, M.L.; Gong, C.; Chen, C.H.; Hu, H.; Huang, P.; Zheng, M.; Yao, Y.; Wei, S.; Wulf, G.; Lieberman, J.; et al. The Rab2A GTPase promotes breast cancer stem cells and tumorigenesis via Erk signaling activation. Cell Rep. 2015, 11, 111-124. [CrossRef]

45. Yang, P.S.; Yin, P.H.; Tseng, L.M.; Yang, C.H.; Hsu, C.Y.; Lee, M.Y.; Horng, C.F.; Chi, C.W. Rab5A is associated with axillary lymph node metastasis in breast cancer patients. Cancer Sci. 2011, 102, 2172-2178. [CrossRef] [PubMed]

46. Frittoli, E.; Palamidessi, A.; Marighetti, P.; Confalonieri, S.; Bianchi, F.; Malinverno, C.; Mazzarol, G.; Viale, G.; Martin-Padura, I.; Garre, M.; et al. A RAB5/RAB4 recycling circuitry induces a proteolytic invasive program and promotes tumor dissemination. J. Cell Biol. 2014, 206, 307-328. [CrossRef] [PubMed]

47. Xie, J.; Yan, Y.; Liu, F.; Kang, H.; Xu, F.; Xiao, W.; Wang, H.; Wang, Y. Knockdown of Rab7a suppresses the proliferation, migration, and xenograft tumor growth of breast cancer cells. Biosci. Rep. 2019, 39. [CrossRef]

48. Yoon, S.O.; Shin, S.; Mercurio, A.M. Hypoxia stimulates carcinoma invasion by stabilizing microtubules and promoting the Rab11 trafficking of the $\alpha 6 \beta 4$ integrin. Cancer Res. 2005, 65, 2761-2769. [CrossRef]

49. Recchi, C.; Seabra, M.C. Novel functions for Rab GTPases in multiple aspects of tumour progression. Biochem. Soc. Trans. 2012, 40, 1398-1403. [CrossRef]

50. Bobrie, A.; Krumeich, S.; Reyal, F.; Recchi, C.; Moita, L.F.; Seabra, M.C.; Ostrowski, M.; Théry, C. Rab27a supports exosome-dependent and -independent mechanisms that modify the tumor microenvironment and can promote tumor progression. Cancer Res. 2012, 72, 4920-4930. [CrossRef]

51. Binenbaum, Y.; Fridman, E.; Yaari, Z.; Milman, N.; Schroeder, A.; Ben David, G.; Shlomi, T.; Gil, Z. Transfer of miRNA in Macrophage-Derived Exosomes Induces Drug Resistance in Pancreatic Adenocarcinoma. Cancer Res. 2018, 78, 5287-5299. [CrossRef]

52. Shen, D.W.; Gottesman, M.M. RAB8 enhances TMEM205-mediated cisplatin resistance. Pharm. Res. 2012, 29, 643-650. [CrossRef]

53. Dorayappan, K.D.P.; Wanner, R.; Wallbillich, J.J.; Saini, U.; Zingarelli, R.; Suarez, A.A.; Cohn, D.E.; Selvendiran, K. Hypoxia-induced exosomes contribute to a more aggressive and chemoresistant ovarian cancer phenotype: A novel mechanism linking STAT3/Rab proteins. Oncogene 2018, 37, 3806-3821. [CrossRef] [PubMed] 
54. Wu, B.; Qi, R.; Liu, X.; Qian, L.; Wu, Z. Rab18 overexpression promotes proliferation and chemoresistance through regulation of mitochondrial function in human gastric cancer. Onco Targets. Ther. 2018, 11, 7805-7820. [CrossRef] [PubMed]

55. Giordano, C.; Gelsomino, L.; Barone, I.; Panza, S.; Augimeri, G.; Bonofiglio, D.; Rovito, D.; Naimo, G.D.; Leggio, A.; Catalano, S.; et al. Leptin Modulates Exosome Biogenesis in Breast Cancer Cells: An Additional Mechanism in Cell-to-Cell Communication. J. Clin. Med. 2019, 8, 1027. [CrossRef] [PubMed]

56. Rovito, D.; Giordano, C.; Plastina, P.; Barone, I.; De Amicis, F.; Mauro, L.; Rizza, P.; Lanzino, M.; Catalano, S.; Bonofiglio, D.; et al. Omega-3 DHA- and EPA-dopamine conjugates induce PPAR $\gamma$-dependent breast cancer cell death through autophagy and apoptosis. Biochim. Biophys. Acta BBA Gen. Subj. 2015, 1850, 2185-2195. [CrossRef]

57. Mauro, L.; Sisci, D.; Bartucci, M.; Salerno, M.; Kim, J.; Tam, T.; Guvakova, M.A.; Ando, S.; Surmacz, E. SHC$\alpha 5 \beta 1$ integrin interactions regulate breast cancer cell adhesion and motility. Exp. Cell Res. 1999, 252, 439-448. [CrossRef]

58. Catalano, S.; Panza, S.; Malivindi, R.; Giordano, C.; Barone, I.; Bossi, G.; Lanzino, M.; Sirianni, R.; Mauro, L.; Sisci, D.; et al. Inhibition of leydig tumor growth by farnesoid $\mathrm{X}$ receptor activation: The in vitro and in vivo basis for a novel therapeutic strategy. Int. J. Cancer 2013, 132, 2237-2247. [CrossRef] [PubMed]

59. Catalano, S.; Malivindi, R.; Giordano, C.; Gu, G.; Panza, S.; Bonofiglio, D.; Lanzino, M.; Sisci, D.; Panno, M.L.; Andò, $\mathrm{S}$. Farnesoid $\mathrm{X}$ receptor, through the binding with steroidogenic factor 1-responsive element, inhibits aromatase expression in tumor Leydig cells. J. Biol. Chem. 2010, 285, 5581-5593. [CrossRef] [PubMed]

60. Giordano, C.; Rovito, D.; Barone, I.; Mancuso, R.; Bonofiglio, D.; Giordano, F.; Catalano, S.; Gabriele, B.; Andò, S. Benzofuran-2-acetic ester derivatives induce apoptosis in breast cancer cells by upregulating p21Cip/WAF1 gene expression in p53-independent manner. DNA Repair 2017, 51, 20-30. [CrossRef] [PubMed]

61. Kulak, N.A.; Pichler, G.; Paron, I.; Nagaraj, N.; Mann, M. Minimal, encapsulated proteomic-sample processing applied to copy-number estimation in eukaryotic cells. Nat. Methods 2014, 11, 319-324. [CrossRef] [PubMed]

62. Rappsilber, J.; Ishihama, Y.; Mann, M. Stop and go extraction tips for matrix-assisted laser desorption/ionization, nanoelectrospray, and LC/MS sample pretreatment in proteomics. Anal. Chem. 2003, 75, 663-670. [CrossRef] [PubMed]

63. Bakunts, A.; Orsi, A.; Vitale, M.; Cattaneo, A.; Lari, F.; Tade, L.; Sitia, R.; Raimondi, A.; Bachi, A.; van Anken, E. Ratiometric sensing of $\mathrm{BiP}$-client versus BiP levels by the unfolded protein response determines its signaling amplitude. Elife 2017, 6. [CrossRef]

64. Cox, J.; Mann, M. MaxQuant enables high peptide identification rates, individualized p.p.b.-range mass accuracies and proteome-wide protein quantification. Nat. Biotechnol. 2008, 26, 1367-1372. [CrossRef]

65. Cox, J.; Neuhauser, N.; Michalski, A.; Scheltema, R.A.; Olsen, J.V.; Mann, M. Andromeda: A peptide search engine integrated into the MaxQuant environment. J. Proteome Res. 2011, 10, 1794-1805. [CrossRef]

66. Steinbichler, T.B.; Dudas, J.; Skvortsov, S.; Ganswindt, U.; Riechelmann, H.; Skvortsova, I.-I. Therapy resistance mediated by exosomes. Mol. Cancer 2019, 18, 58. [CrossRef]

(C) 2020 by the authors. Licensee MDPI, Basel, Switzerland. This article is an open access article distributed under the terms and conditions of the Creative Commons Attribution (CC BY) license (http://creativecommons.org/licenses/by/4.0/). 